\title{
Application of Fuzzy Expert System in Determination of MMPI-2 Protocol Code Type
}

\author{
Pavel Škobrtal1, Iveta Bebčáková2 ${ }^{2}$ Jana Talašová ${ }^{2}$
}

${ }^{1}$ Department of Pedagogical and School Psychology, University of Ostrava

${ }^{2}$ Department of Mathematical Analysis and Applications of Mathematics, Palacký University Olomouc

Correspondence concerning this article should be addressed to Pavel Škobrtal, Department of Pedagogical and School Psychol-

ogy, University of Ostrava, Dvořákova 7, 70103 Ostrava, Czech Republic. E-mail: pavel.skobrtal@email.cz

\begin{abstract}
The MMPI-2 questionnaire is one of the key methods used for detection of significant personality features and mental disorders. It is a complex questionnaire and its interpretation includes two parts, quantitative and qualitative one. The crucial part of the quantitative interpretation is determination of a code type of the given protocol. In our article, we introduce a mathematical model for determination of protocol's code type, which, in addition to clinical scales, takes into consideration results of the other scales according to the original interpretation manual for MMPI-2. The model is based on a linguistically defined base of rules. By using the base of rules, the model is also able to detect prototypic protocols which correspond to the administered protocol the best.
\end{abstract}

\section{Application of Fuzzy Expert System in Determination of MMPI-2 Protocol Code Type}

MMPI-2 questionnaire (Minnesota Multiphasic Personality Inventory 2) belongs to the three most used psychodiagnostic methods in the world (Greene, 2002). The questionnaire serves for testing of personality features and mental disorders. It is especially used in clinical practice. The questionnaire was created on the base of revision of the original MMPI questionnaire which was published by S. Hathaway and J. C. McKinley in 1940 (Butcher, Graham, Williams, 1990). The questionnaire consists of 567 questions in total. The original version contains 10 clinical scales Hypochondria (Hs), Depression (D), Conversion Hysteria (Hy), Psychopathic Deviation (Pd), Masculinity-Feminity (Mf), Paranoia (Pa), Psychasthenia (Pt), Schizophrenia (Sc), Hypomania (Ma), Social introversion (Si) and standard validity scales. If compared with that, the other scales were introduced later as for example Harris-Lingoes Subscales, Si Subscales, content scales, Wiener-Harmon Subscales, Personality Psychopathology Scales, Behavioural Dysfunction Scales and supplemental scales (Netík, 2002).

The questionnaire might be administered either individually or in groups. The administration takes for about 1 up to 2 hours. The base of questionnaire interpretation is determination of a code type for the protocol. There are 55 code types in total. The code type is determined by one or two clinical scales with the highest $\mathrm{T}$-score that is higher than 65 . We refer to the code type by recording of the numbers of these two scales. At the same time, the higher scale is recorded at first. If both the scales have the same value, they are stated in numerical order. In a case when there is only one clinical scale in the protocol higher than $65 \mathrm{~T}$-scores, we talk about so called spiky profile. We work with most types independently on the order of the scales in code type. However, there are exceptions when the interpretation of protocol is directly influenced by the order of scales of the code type. For the code type interpretation there are many systems created. The code type that has been defined well is a code type which value of T-score for one or two clinical scales has higher value than 65 and the T-score is at least by 5 points higher than the Tscore of the other clinical scales. In the opposite case, it rather deals with a triad and the code type cannot be used for interpretation. About two thirds of the codes are not defined well (Greene, 2002; Archer, 2005).

In order that it was possible to proceed from characteristics of the appropriate code type for interpretation, at first patient's protocol must be compared with a prototypic protocol of the given code type. For analysis, it is necessary in order that the code type was defined well. Prototypic protocols contain scores for all clinical, content, supplemental and other scales that are standardly scored. In prototypic protocols all the scores are stated in T-scores with the appropriate K-correction with the exception of "?" scale that is stated in rough scores. If a score or scores of the obtained protocol vary more than by one standard deviation (that is by $10 \mathrm{~T}$-scores) from the scores of prototypic protocol of the appropriate code type, the code type characteristics cannot be used completely. If the code type has been defined pseudo-well, we may proceed from the interpretation scheme for the given code type. However, we must keep in mind that the interpretation of the given code type serves as a certain frame only for interpretation of the other scales and cannot be complied with mechanically. However, more complicated situation will occur if it deals with the code type that has been defined pseudo-badly. Then it must be decided which code type to use as a starting frame for interpretation of the given protocol.

As regards the questionnaire evaluation, Testcentrum Praha Company Ltd. developed computer software MPI-2 v5.27. This software which is delivered with the questionnaire defines the code type of protocol on the base of two highest clinical scales. However, the software doesn't take the other scales into account and because of that the interpretation of the protocol may not be quite precise on many occasions. The aim of our work was to develop a mathematical model that would also take results of the other scales of the questionnaire into account during determination of the code type which would better corresponded to requirements of the original manual.

\section{Method}

During psychodiagnostic examination, we often meet problems that we have to evaluate and interpret data which we obtained after application of testing or clinical methods by a suitable way. At the same time, the data in psychology are not a result of direct measurements (by means of special instruments and devices) as we 
know that from sciences. Because of the fact that many human characteristics and features cannot be precisely measured thanks to their nature, psychologists must often use vague concepts which are offered by natural language.

One from the methods that is used for processing of vague resp. inaccurate data is the Theory of Fuzzy Sets (Smithson, Verkuilen, 2006). This theory offers mathematical apparatus that manages to work with such blurry values. A basic idea of fuzzy sets could be expressed by the following words: "If we are not able to determine precise boundaries of a class delimited by a vague concept, we have to substitute a definite decision on membership or non-membership of every element in this class by a rate from interval $<0,1>$ that describes a grade of its membership in the given class. Lower value will indicate that the given element lies somewhere at the edge of the class and opposite. This value is called a grade of membership of the given element to the given class. The class in which every element has been characterized by a grade of membership to this class is called a fuzzy set (Zadeh, 1965).

A special case of a fuzzy set is a fuzzy number. In this case, considered elements are real numbers. In Picture No. 1 there is an example of the simplest fuzzy number shown. It models the concept of "Considerably Increased T-score". It is obvious from the picture that values higher then 65 are considered with a certainty to be considerably increased (the grade of membership is equal to one) while T-scores lower than 63 are not considerably increased with a certainty (the grade of membership of such elements is equal to zero). The area between these values is encumbered by uncertainty. Transition between absolute membership and absolute non-membership is defined linearly.

Fuzzy sets theory means also a good tool for modelling of values of linguistic variables coming from the natural language and for creation of defined bases of linguistic rules (Novák, 1990). We understand a linguistic variable the variable which takes words. Senses of these words are mathematically modelled in fuzzy sets (Novák, 1990; Talašová, 2003). An example of the linguistic variable might be Tscore of some examined scale. Its values are linguistic terms "Unimportant" and "Considerably Increased" which are modelled by means of fuzzy numbers in Picture No. 2.

Relation between linguistic variables might be expressed by linguistically defined function (base of rules). It deals with a system of linguistically defined rules of the type of "If- then" that enable description of a complicated relation between input and output linguistic variables, e.g. "If T-score of the most increased scale is considerably increased, T-score of the second increased scale is unimportant and the difference between the first and second scale is big enough, then it deals with a spiky code type". These rules are defined expertly, respectively obtained from well-known data.

Process of substituting of monitored linguistic values of the input linguistic variables into the base of rules and looking for corresponding linguistic values of the output linguistic variable is indicated as approximate deduction. There are several various methods of approximate deduction. One from the well-known and most often used methods is Mamdani Method (Talašová, 2003). The base of rules together with the used algorithm of approximate deduction creates so called Fuzzy Expert System (Dubois, Prade, 2000).

\section{Results}

We used the fuzzy sets theory for creation of a mathematical model which, on the base of MMPI-2 questionnaire, defines a code type. In addition, it offers information on the fact which further prototypic protocols are the most similar to the tested protocol. By that, a psychologist is given not only information on how many percents the given protocol agrees with the prototypic protocol but also the information on the other similar protocols. By that, the psychologist is given more information for consequent clinical consideration.

The proposed mathematical model consists of the two fuzzy expert systems. The first system tries to indicate a code type of the protocol. The second one makes a test on compliance of the tested protocol with prototypic protocols.

In a case of indication of protocols code type, there are ten main clinical scales put in order on the base of obtained T-score from the highest score to the lowest score. Further, we defined 6 linguistic variables: T-score of the first highest scale, T-score of the second highest scale, T-score of the third highest scale, a difference between the first and second highest scale, a difference between the second and the third highest scale and a shape of code type. On the base of these 6 linguistic variables and available methodology of code type identification, we constructed a base of rules. The base is created by 4 rules. Every rule describes one from possibilities; that is when it deals with a spiky code type, a pair, a triad or non-pathological protocol. The values of previously ordered clinical scales of the tested protocol are substituted into the base of rules that was constructed that way. The base of rules with utilisation of Mamdani Method of approximate deduction will indicate the code type that it deals with (See an example in chapter Results).

In the second phase, our model compares the scores of the given questionnaire with the scores of prototypic protocols. Every prototypic protocol was described in a simplified fashion by means of 79 input linguistic variables which represent T-scores achieved in the individual scales. Every input linguistic variable will assume four linguistic values. Low, Normal, Lightly Increased and Extremely Increased, see Picture No. 3.

55 rules were created by utilisation of the linguistic variables defined this way. Every rule corresponds to one from 55 prototypic protocols, e.g. "If T-score of ? scale is low and T-score of $L$ scale is low and ... and T-score of APS scale is normal then it deals with a prototypic protocol of the code type 1." Together with adjusted Mamdani algorithm for approximate deduction, the base of rules defined this way created the second expert system of our mathematical model (Bebčáková, Talašová, Škobrtal 2009).

We will demonstrate model behaviour on an example of analysis of one from the tested protocols. On the base of questionnaire, T-scores illustrated in Graph No. 1 were assigned to the individual clinical scales. The original program analysed the given protocol as the code type 2-7/7-2.

A possibility that it deals with the type of 2-7/7-2 was evaluated by number 0.8 in our software. A possibility to interpret the given protocol as a triad was evaluated by value of 0.2 (See Graph No. 2). So, our mathematical model responded to the fact that the difference between the second highest and third highest scale is lower than required $5 \mathrm{~T}$-scores, by reduction of possibility measure that it deals with a pair. 
The three prototypic protocols that show the highest compliance with the tested protocol were indicated by our program in the following way:

Type 6886 with compliance rate of 0.496203 ,

Type 7887 with compliance rate of 0.470886 ,

Type 2882 with compliance rate of 0.458228 ,

From Graph No. 3 it is obvious that there is a deviation of several tenths between the three most similar prototypic protocols and the prototypic protocol of code type 2-7/7-2. So, we may proceed from the code type of 2-7/7-2 during interpretation with a certain reserve.

\section{Discussion}

Our paper monitored utilisation of fuzzy sets during interpretation of MMPI-2 questionnaire results. In concrete terms, we focused on a process of indication of the code type on the base of which the protocol was interpreted further. For this purpose, we decided to create a mathematical model that would indicate the code type that it dealt with in the first phase. Later, it should have evaluated whether the given protocol corresponded to prototypical scores for this code type and at the same time to show information on the fact which prototypical protocols were the nearest to our protocol. Here, we encountered a problem. During construction of the mathematical model, we created the individual linguistic variables on the base of individual scales that were parts of MMPI-2 questionnaire. Not all the scales which are a part of the questionnaire were translated into Czech version. Further, Czech prototypic protocols have not been created yet. That is why we had to proceed from American prototypic protocols. From that reason we used 79 scales only from the total number of all the scales stated in prototypic protocols. We made comparison of patients' protocols with these prototypic protocols. In our model, we did not treat aggravation or trivialization of psychopathological symptoms by any way. We did not reflect results of factor scales either or similar. This simplification was based on the fact that the MMPI-2 questionnaire is very complicated with its many scales and indexes. From that reason we decided to start working with the simplified model that would have been developed and deepened consequently. We think that our model is utilisable even if it has been simplified. However, the circumstances stated above must be considered while using it. For the time being, the model is being developed and improved.

\section{Conclusion}

Our paper is concerned with a possibility of application of the fuzzy expert system for identification of a code type from MMPI-2 questionnaire. The way described above was used for analysis of 100 protocols of psychiatric patients selected at random. We found out different conclusion in comparison with the original software in 26 protocols from 100 as regards identification of the code type (the original software indicates the code type on the base of the two highest clinical scales only). If it deals with comparison of the given protocol with a prototypic protocol of the appropriate code type, the results which we obtained must be interpreted carefully from the reasons stated above. We think that the results of our work may be considered highly valuable for clinical area of psychology especially for diagnostics and they should be processed further.

\section{References}

Archer, R. P. (2005). MMPI-A: Assessing adolescent psychopathology. New York: Lawrence Erlbaum Associates.

Bebčáková, I., Talašová, J., \& Škobrtal, P. (2009). Fuzzy Approach to Quantitative Interpretation of MMPI-2. Journal of Applied Mathematics, 1, 65-75.

Butcher, J. N., Graham, J. R., Williams, C. L., \& Ben-Porath, Y. S. (1990). Development and use of the MMPI-2 content scales. Minnesota: University of Minnesota Press.

Dubois, D., \& Prade, H. (2000). Fundamentals of Fuzzy Sets. Norwell: Kluwer Academic Publishers.

Greene, R. L. (2002). The MMPI-2: An interpretative manual. Boston: Allyn \& Bacon.

Netík, K. (2002). MMPI-2 Minnesota Multiphasic Personality Inventory - 2. Praha: Testcentrum.

Novák, V. (1990). Fuzzy Sets and Their Application. Praha: SNTL.

Smithson, M., \& Verkuilen, J. (2006). Fuzzy Set Theory: Application in the Social Sciences. Thousand Oaks: Sage Publications, Inc.

Svoboda, M. (1999). Psychological Diagnostics of Adults. Praha: Portál.

Talašová, J. (2003). Fuzzy Methods of Multicriterial Evaluation and Decision Making. Olomouc: Univerzita Palackého v Olomouci.

Zadeh, L. A. (1965). Fuzzy Sets. Information and Control, 8, 338-353. 
Figure 1. Fuzzy Number

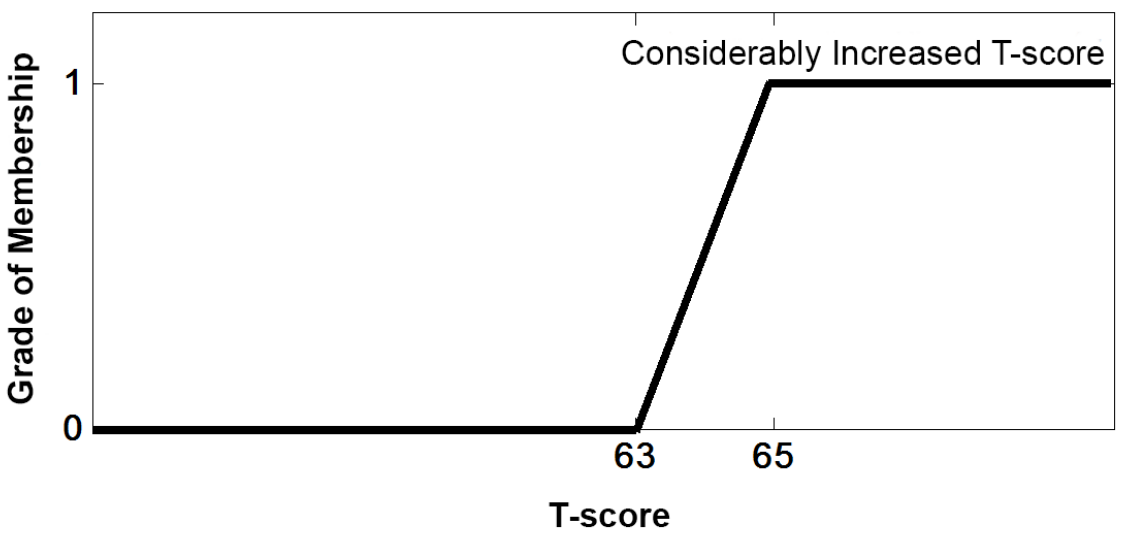

Figure 2. Linguistic Variable

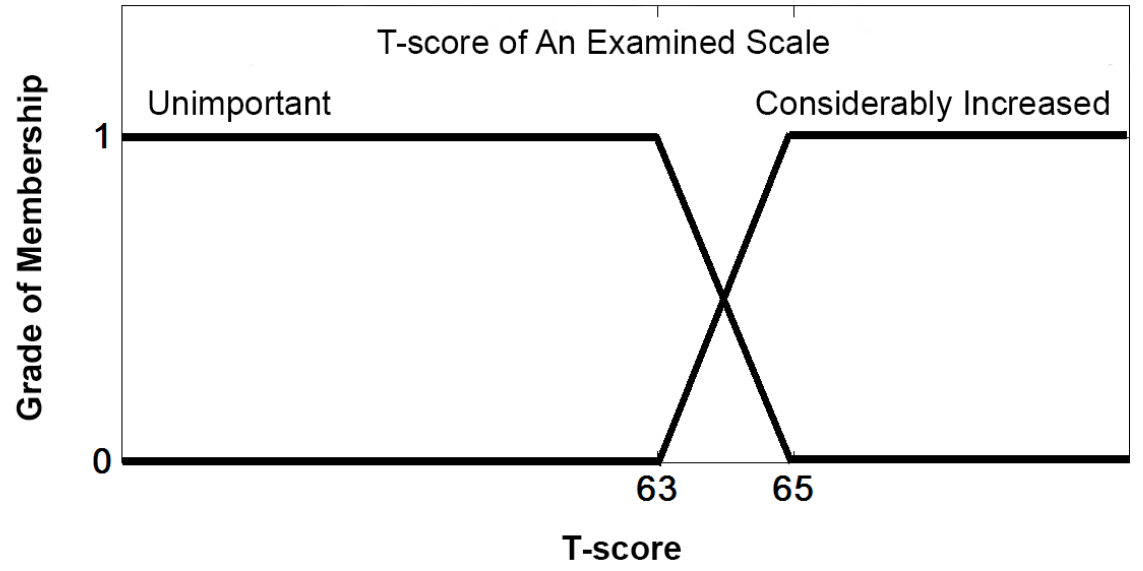


Figure 3. Fuzzy Numbers Modelling Senses of Values of Input Linguistic Variable T-score

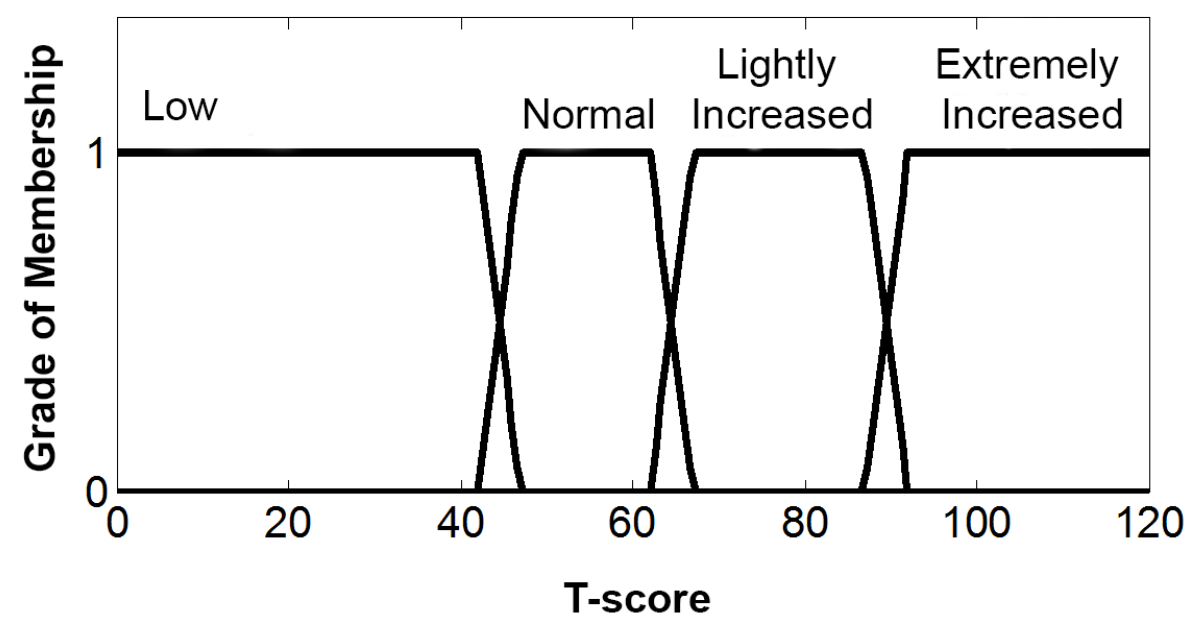

Figure 4. Clinical Scales Scores

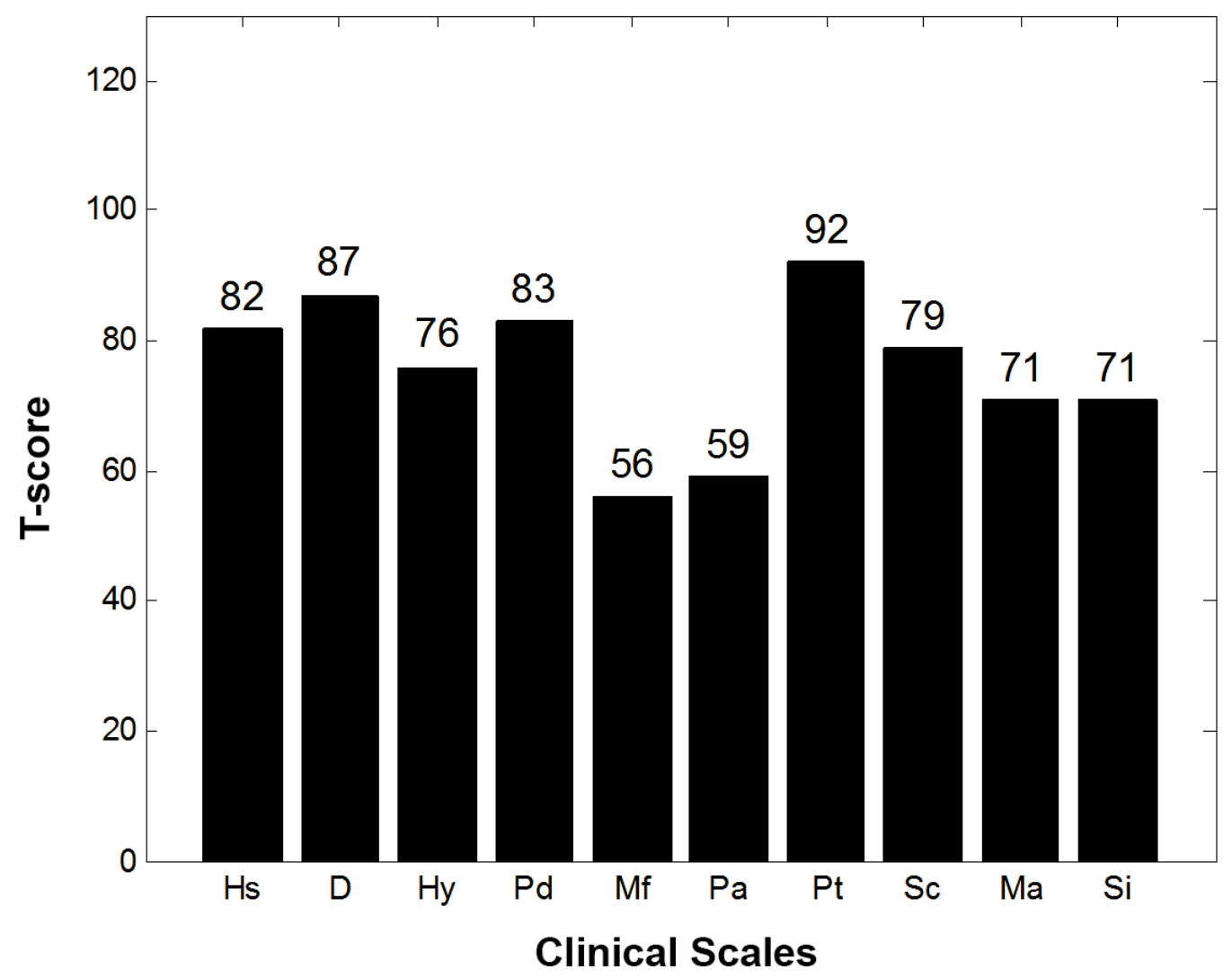




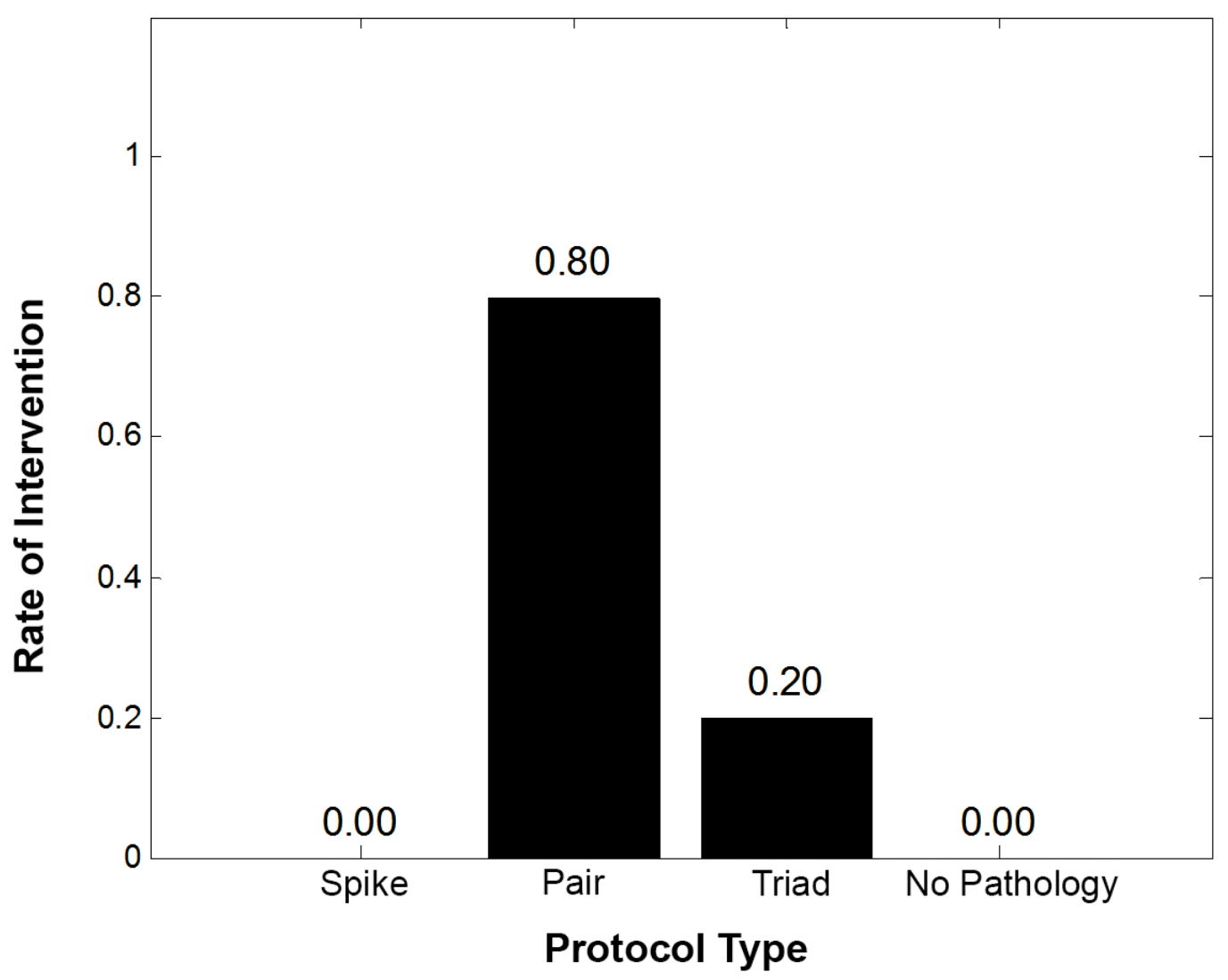

Figure 6. Comparison with Prototypic Protocols

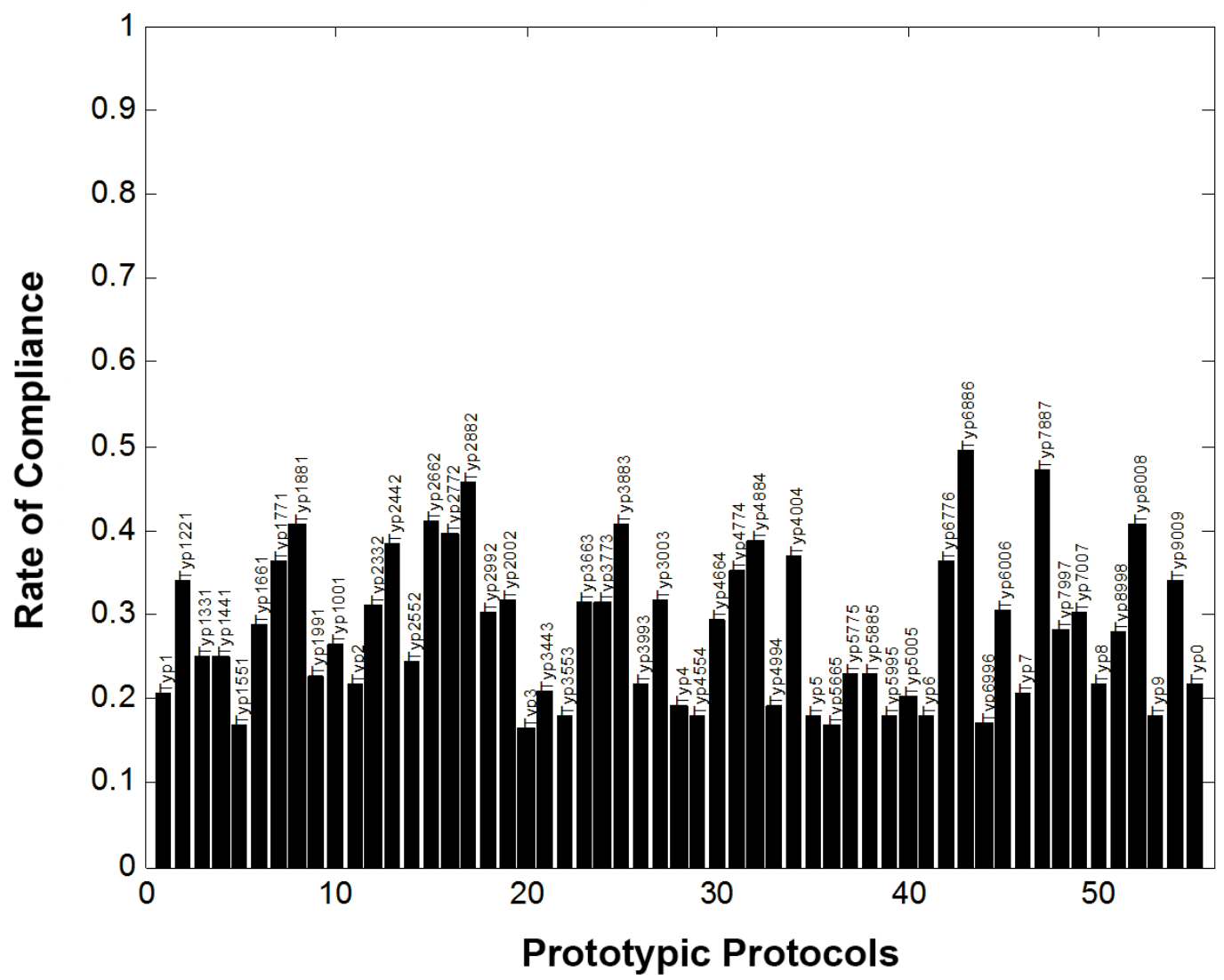

